\title{
Gestational Trophoblastic Neoplasia with Gum Metastasis: A Case Study and Literature Review
}

\author{
Nida Jareemit Mongkol Benjapibal \\ Division of Gynecologic Oncology, Department of Obstetrics and Gynecology, Faculty of \\ Medicine Siriraj Hospital, Mahidol University, Bangkok, Thailand
}

\section{Keywords}

Gestational trophoblastic neoplasia · Gum metastasis · High risk

\begin{abstract}
Gestational trophoblastic neoplasia (GTN) is an uncommon group of pregnancy-related malignancies. Delayed diagnosis is a prognostic factor for worse outcome. GTN is even harder to diagnose if the site of metastasis is uncommon. The reported case is a 27-year-old $G_{2} P_{1} A_{1}$ woman who presented to our center with acute transient generalized tonic-clonic seizure. She had developed hemoptysis for the 2 preceding weeks, which had been treated as pneumonia. She had then noticed multiple gum lesions and vaginal spotting 1 week before her presentation. Her serum $\beta$-human chorionic gonadotropin level was 77,474 IU/L without evidence of pregnancy. She was diagnosed with GTN with lung, brain, and gum metastases. The patient was staged as IV with a World Health Organization prognostic score of 14. Etoposide, methotrexate, actinomycin D alternating with cyclophosphamide, and vincristine weekly (EMACO) were given. The gum lesions disappeared after 2 cycles of the multiagent chemotherapy, and complete remission was achieved after 8 cycles. This case study will increase awareness of uncommon metastatic sites of GTN. Any associated vaginal bleeding should be considered a clue to metastatic GTN.

\section{Introduction}

Gestational trophoblastic neoplasia (GTN) denotes a rare group of pregnancy-related malignancies that are caused by abnormal proliferation of placental trophoblasts. GTN is an uncommon cancer, but it responds to chemotherapy very well, resulting in a high survival rate. 
Similar to all cancers, early diagnosis followed by immediate and appropriate treatment yields the best outcomes; however, histopathological confirmation of GTN is not essential. A clinical diagnosis is typically made based on an abnormal pattern of serum $\beta$-human chorionic gonadotropin ( $\beta$-hCG) during molar surveillance. Difficulties arise when patients present with symptoms of metastasis after a nonmolar antecedent pregnancy. In that case, GTN was diagnosed due to a rise in serum $\beta$-hCG without any evidence of pregnancy. It is even harder to diagnose GTN if the site of metastasis is uncommon.

Here, we report on a rare case of ultra-high-risk GTN with atypical gingival metastasis in which the gum lesions demonstrated a rapid response to multiagent chemotherapy. A literature review of studies on GTN with gum metastasis is also presented.

\section{Case Report}

A 27-year-old $\mathrm{G}_{2} \mathrm{P}_{1} \mathrm{~A}_{1}$ woman had developed transient alteration of consciousness and generalized tonic-clonic seizure $1 \mathrm{~h}$ prior to her emergency arrival at our center. Two weeks earlier, she had had hemoptysis with low-grade fever. Chest radiography (chest X-ray) at her primary care hospital had revealed a $3.1 \times 4.6 \times 5.0 \mathrm{~cm}$ mass at the lower lobe of her left lung. Pneumonia had been diagnosed and treated with intravenous antibiotics. She had then been lost to follow-up due to improvement in her symptoms. One week later, the patient had noticed multiple gum lesions and vaginal spotting.

The patient had one child by normal delivery 5 years ago, and a molar pregnancy 1 year ago. The last serum $\beta$-hCG surveillance had been performed 3 months after suction curettage with the level of approximately $400 \mathrm{IU} / \mathrm{L}$. She had then been lost to follow-up. She had experienced amenorrhea until the last week that vaginal spotting had occurred. She had previously been healthy and sexually active with no contraception use.

Upon arrival at our center, the patient appeared slightly drowsy. Her Glasgow Coma Scale was $\mathrm{E}_{3} \mathrm{~V}_{4} \mathrm{M}_{5}$, and no sign of neurological deficit was detected. Her vital signs were normal. She had mildly pale conjunctivae and no jaundice. She had two 7-mm purplish nodules at the outer and inner aspects of her gingiva between the upper incisors, and three 4-mm erythematous lesions around her left upper molar teeth (Fig. 1). Her chest, cardiac, and abdominal examination results were normal. Pelvic examination was unremarkable, except for a small amount of blood in the vagina.

A urine pregnancy test was positive. Transvaginal ultrasonography revealed a normalsized uterus. The endometrial thickess was $3 \mathrm{~mm}$. Neither an intrauterine gestational sac nor content was observed. Both ovaries were unremarkable. Her serum $\beta$-hCG level was 77,474 IU/L; thus, postmolar GTN was suspected. A metastatic survey consisting of chest X-ray and brain and abdominal computed tomography was performed. Abnormal findings included a $5-\mathrm{cm}$ mass at the left lung, a $3-\mathrm{cm}$ well-defined nonenhancing lesion at the right occipital lobe, and another $0.8-\mathrm{cm}$ well-defined nonenhancing lesion in the right parietal region. Acute subdural hemorrhage and a $0.8-\mathrm{cm}$ midline shift were also noted. As a result, the patient was staged as IV with a World Health Organization prognostic score of 14 ( 4 for an interval >12 months from index pregnancy, 2 for the level of serum $\beta$-hCG, 2 for the largest tumor size at the left lung, 4 for brain metastasis, and 2 for eight metastatic lesions).

Intravenous mannitol, dexamethasone, and Dilantin were initially given to treat the cerebral edema and to prevent subsequent seizure. Etoposide, methotrexate, actinomycin D alternating with cyclophosphamide, and vincristine weekly (EMACO) were given. The patient had a dramatic response to the treatment, evidenced by resolution of the gum and lung lesions after 2 and 4 cycles of chemotherapy, respectively. She achieved normalization of her serum 


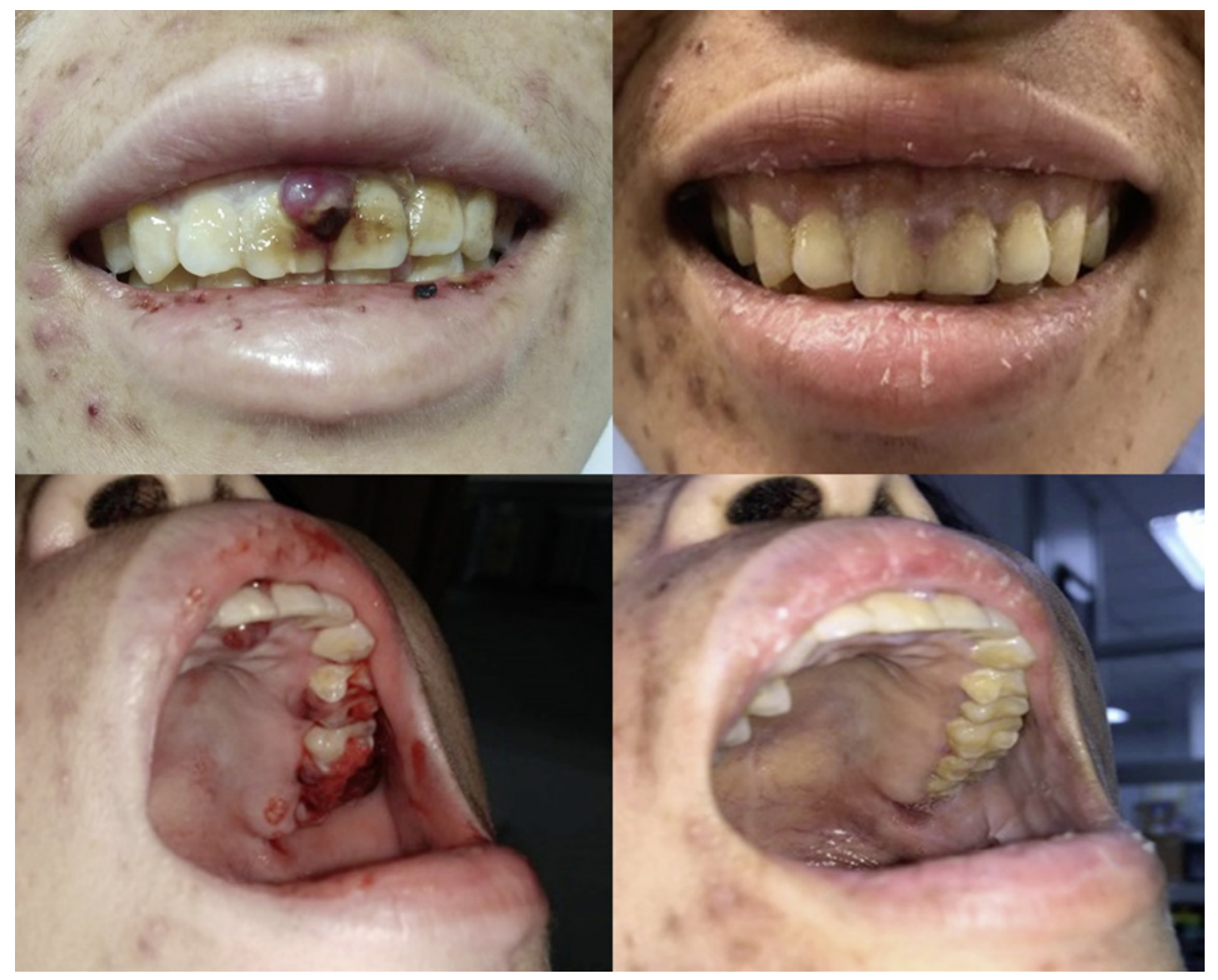

Fig. 1. Multiple purplish nodules at the upper incisors and left upper molar teeth before (left column) and after (right column) receiving 2 courses of chemotherapy.

$\beta$-hCG level following 5 cycles of chemotherapy and 3 consolidation cycles. Currently, the patient is being surveilled, and has been remaining in complete remission after 11 months of follow-up.

\section{Discussion}

GTN is a curable cancer. Complete remission can be achieved in almost all low-risk GTN patients receiving single-agent chemotherapy, while combined chemotherapy can yield a complete remission rate of up to $90 \%$ among high-risk patients [1-3]. Prognostic factors related to treatment failure include a histological diagnosis of choriocarcinoma, liver or brain metastasis, delayed diagnosis, and inappropriate treatment [1,4]. Importantly, some of these risk factors are modifiable, such as delayed diagnosis. A diagnosis of GTN should be considered for patients with a past history of pregnancy, especially molar pregnancy, who present with a missed menstrual period or abnormal uterine bleeding and metastatic symptoms such as dyspnea, tachypnea, and/or seizure.

The most common metastatic sites of GTN are the lung (80\%), vagina (30\%), pelvis (20\%), liver (10\%), and brain (10\%) [5]. The common presentations should be taken into account, and uncommon symptoms should be reported. New finding updates will improve 
Table 1. Literature review of studies on GTN patients with gum metastasis

\begin{tabular}{|c|c|c|c|c|c|c|c|}
\hline $\begin{array}{l}\text { Study } \\
\text { [Ref.], year }\end{array}$ & $\begin{array}{l}\text { Age, } \\
\text { years }\end{array}$ & Chief complaint & Gum lesions & Metastatic sites & Gum biopsy & Chemotherapy & Outcome \\
\hline $\begin{array}{l}\text { Altintas et al. } \\
{[6], 1995}\end{array}$ & 26 & $\begin{array}{l}\text { Painful gingival } \\
\text { mass }\end{array}$ & $\begin{array}{l}\text { Painful mass in } \\
\text { maxillary gingiva }\end{array}$ & Lung & $\begin{array}{l}\text { Yes, } \\
\text { choriocarcinoma }\end{array}$ & MAC, 6 cycles & Remission \\
\hline $\begin{array}{l}\text { Shafiee et al. } \\
\text { [7], } 2011\end{array}$ & 23 & $\begin{array}{l}\text { Alteration of } \\
\text { consciousness }\end{array}$ & $\begin{array}{l}\text { Ulcerative and bleeding } \\
\text { mass in left upper gum }\end{array}$ & $\begin{array}{l}\text { Lung, brain, liver, } \\
\text { spleen, kidney, } \\
\text { and peritoneum }\end{array}$ & $\begin{array}{l}\text { Yes, } \\
\text { choriocarcinoma }\end{array}$ & No chemotherapy & $\begin{array}{l}\text { Disease- } \\
\text { related } \\
\text { death }\end{array}$ \\
\hline $\begin{array}{l}\text { Alifrangis et } \\
\text { al. [8], } 2011\end{array}$ & 46 & Toothache & $\begin{array}{l}\text { Painful and bleeding } \\
\text { vascular lesion in left } \\
\text { upper gum }\end{array}$ & $\begin{array}{l}\text { Lung, brain, liver, } \\
\text { and kidney }\end{array}$ & No & $\begin{array}{l}\text { EMACO, then TPTE } \\
\text { with intrathecal } \\
\text { methotrexate }\end{array}$ & Remission \\
\hline Present case & 27 & $\begin{array}{l}\text { Alteration of } \\
\text { consciousness }\end{array}$ & $\begin{array}{l}\text { Asymptomatic purplish } \\
\text { gingival nodules in the } \\
\text { upper incisor and molar } \\
\text { area }\end{array}$ & Lung and brain & No & EMACO, 8 cycles & Remission \\
\hline
\end{tabular}

GTN, gestational trophoblastic neoplasia; MAC, methotrexate, actinomycin D, and cyclophosphamide; EMACO, etoposide, methotrexate, actinomycin D, cyclophosphamide, and vincristine.

diagnostic timeliness and accuracy and also improve the outcomes. The recognition of unusual metastatic lesions as being associated with GTN will help avoid harmful biopsy of this hypervascularized tumor, which may cause profuse and potentially lethal bleeding. Fortunately, a histopathological diagnosis is not essential for the diagnosis and treatment of GTN. However, and of concern, an inaccurate evaluation of metastatic lesions can lead to inappropriate chemotherapeutic regimens. For example, single-agent chemotherapy, which should be given in cases of low-risk GTN, may be incorrectly prescribed in cases of high-risk GTN instead of a multiagent regimen.

Very few studies have reported metastatic gum lesions from GTN. These lesions are painful and purplish in color, and they bleed easily upon contact. However, these features are nonspecific and sometimes are difficult to distinguish from common benign gingival diseases such as benign pyogenic granuloma, peripheral giant cell granuloma, or peripheral ossifying granuloma [6-8].

Gum metastasis in patients with GTN is extremely rare, accounting for less than $1 \%$ of all oral malignancies. In female patients, the most common primary malignancies are of the breast,genital organs, bone, and kidney [9]. If GTN with gum metastasis is highly suspected, such as in our case, we do not recommend performing any biopsies due to the risk of massive hemorrhage. Instead, chemotherapy is prescribed with subsequent reevaluation of the gum lesions. The response to chemotherapy supports the diagnosis of GTN.

The primary standard treatment for high-risk GTN is multiagent chemotherapy. EMACO is the most commonly used regimen, with a complete response rate of $80-90 \%[1-3,10,11]$. However, in ultra-high-risk GTN, defined as a prognostic score of $\geq 12$, alternative treatment can be etoposide and cisplatin alternating weekly with etoposide, methotrexate, and actinomycin D (EP/EMA), or induction with weekly cisplatin/etoposide for a few weeks prior to conventional multiagent chemotherapy, which was shown to effectuate a significant decline in early death rates due to severe side effects of full-dose chemotherapy [12].

Similar to our patient, previously reported cases were classified as high-risk GTN with multiple organ metastases such as to the lung or brain (Table 1). In 2 of these 3 cases, the patients received combined chemotherapy and achieved complete remission $[6,8]$. One patient had very advanced disease involving the lung, brain, liver, spleen, kidneys, peri- 
toneum, and gingiva. That patient expired before initiation of chemotherapy due to severe coagulopathy and multiple organ failure [7].

Organ-specific treatment is usually not required. Local excision, radiation, or embolization is sometimes employed to relieve localized symptoms or to treat resistant lesions. Our patient presented with brain metastasis and increased intracranial pressure. We initially treated the cerebral edema with mannitol and dexamethasone, followed by EMACO administration. Dramatic improvement in symptoms was demonstrated, as well as in serum $\beta$-hCG levels and follow-up imaging results. Whole brain irradiation was not required, due to our patient's rapid and dramatic response to treatment. To overcome the blood-brain barrier, different strategies have been adopted to determine the adequate therapy for brain tissue, which has resulted in higher remission rates. Examples of these strategies are: increasing the dose of methotrexate to $1 \mathrm{~g} / \mathrm{m}^{2}$ in EMACO, intrathecal methotrexate, combination chemotherapy and whole brain irradiation, or craniotomy with or without tumor resection $[1,13]$. Based on metastatic gum cancer data, good oral hygiene should be promoted, since chronic inflammation from poor oral hygiene may contribute to various processes in tumorigenesis [9].

\section{Statement of Ethics}

This case study was exempted from institutional review board review, and our patient provided written informed consent, permitting us to disclose details relating to her case.

\section{Disclosure Statement}

There are no conflicts of interest to declare.

\section{Funding Sources}

The authors did not receive any funding.

\section{Author Contributions}

N. Jareemit: data collection, literature review, and writing of the original draft; M. Benjapibal: conceptualization, writing of the review, and editing. Both authors read and approved the final version of the manuscript.

\section{References}

1 Ngan HYS, Seckl MJ, Berkowitz RS, Xiang Y, Golfier F, Sekharan PK, et al. Update on the diagnosis and management of gestational trophoblastic disease. Int J Gynaecol Obstet. 2018;143(Suppl 2):79-85.

2 Cagayan MS. High-risk metastatic gestational trophoblastic neoplasia. Primary management with EMA-CO (etoposide, methotrexate, actinomycin D, cyclophosphamide and vincristine) chemotherapy. J Reprod Med. 2012;57(5-6):231-6.

3 Berkowitz RS, Goldstein DP, Horowitz NS. Initial management of high-risk gestational trophoblastic neoplasia. In: Goff B, Dizon DS, editors. UpToDate [Internet]. Wolters Kluwer [updated 2019 Nov 12].

4 Gueye M, Ndiaye-Gueye MD, Kane Gueye SM, Moreau JC. Fatal cases of gestational trophoblastic neoplasia in a national trophoblastic disease reference center in Dakar Senegal. Int J MCH AIDS. 2016;5(1):32-8. 
5 Thomakos N, Rodolakis A, Belitsos P, Zagouri F, Chatzinikolaou I, Dimopoulos AM, et al. Gestational trophoblastic neoplasia with retroperitoneal metastases: a fatal complication. World J Surg Oncol. 2010;8:114.

6 Altintas A, Vardar MA, Aridoğan N, Doran F, Tuncer I. Choriocarcinoma metastatic to the maxillary gingiva. Eur J Surg Oncol. 1995;21(5):579-80.

7 Shafiee MN, Ismail NM, Shan LP, Kampan N, Omar MH, Dali HM. A case report: metastatic choriocarcinoma to the gum. Sex Reprod Healthc. 2011;2(2):91-2.

8 Alifrangis C, Evans R, Williams J, Seckl MJ. An unusual gum lesion with a positive pregnancy test. BMJ. 2011; 343:d5009.

9 Kumar G, Manjunatha B. Metastatic tumors to the jaws and oral cavity. J Oral Maxillofac Pathol. 2013;17(1): 71-5.

10 Deng L, Zhang J, Wu T, Lawrie TA. Combination chemotherapy for primary treatment of high-risk gestational trophoblastic tumour. Cochrane Database Syst Rev. 2013;(1):CD005196.

11 Cyriac S, Rajendranath R, Sridevi V, Sagar TG. Etoposide, cisplatin-etoposide, methotrexate, actinomycin-D as primary treatment for management of very-high-risk gestational trophoblastic neoplasia. Int J Gynaecol Obstet. 2011;115(1):37-9.

12 Alifrangis C, Agarwal R, Short D, Fisher RA, Sebire NJ, Harvey R, et al. EMA/CO for high-risk gestational trophoblastic neoplasia: good outcomes with induction low-dose etoposide-cisplatin and genetic analysis. J Clin Oncol. 2013;31(2):280-6.

13 Cagayan MS, Lu-Lasala LR. Management of gestational trophoblastic neoplasia with metastasis to the central nervous system: a 12-year review at the Philippine General Hospital. J Reprod Med. 2006;51(10):785-92. 